\title{
National single-step genomic method that integrates multi-national genomic information
}

\author{
J. Vandenplas, ${ }^{*} \dagger^{1,2}$ M. Spehar, $¥ \S$ K. Potocnik,§ N. Gengler, ${ }^{*}$ and G. Gorjanc§\# \\ *Agriculture, Bio-engineering and Chemistry Department, Gembloux Agro-Bio Tech, University of Liege, 5030 Gembloux, Belgium \\ †National Fund for Scientific Research, 1000 Brussels, Belgium \\ $\ddagger$ Croatian Agricultural Agency, 10000 Zagreb, Croatia \\ §Biotechnical Faculty, University of Ljubljana, 1000 Ljubljana, Slovenia \\ \#The Roslin Institute and Royal (Dick) School of Veterinary Studies, The University of Edinburgh, Easter Bush Research Centre, \\ Midlothian EH25 9RG, United Kingdom
}

\section{ABSTRACT}

The aim of this paper was to develop a national singlestep genomic BLUP that integrates multi-national genomic estimated breeding values (EBV) and associated reliabilities without double counting dependent data contributions from the different evaluations. Simultaneous use of all data, including phenotypes, pedigree, and genotypes, is a condition to obtain unbiased EBV. However, this condition is not always fully met, mainly due to unavailability of foreign raw data for imported animals. In dairy cattle genetic evaluations, this issue is traditionally tackled through the multiple across-country evaluation (MACE) of sires, performed by Interbull Centre (Uppsala, Sweden). Multiple across-country evaluation regresses all the available national information onto a joint pedigree to obtain country-specific rankings of all sires without sharing the raw data. In the context of genomic selection, the issue is handled by exchanging sire genotypes and by using MACE information (i.e., MACE EBV and reliabilities), as a valuable source of "phenotypic" data. Although all the available data are considered, these "multi-national" genomic evaluations use multi-step methods assuming independence of various sources of information, which is not met in all situations. We developed a method that handles this by single-step genomic evaluation that jointly (1) uses national phenotypic, genomic, and pedigree data; (2) uses multi-national genomic information; and (3) avoids double counting dependent data contributions from an animal's own records and relatives' records. The method was demonstrated by integrating multi-national genomic EBV and reliabilities of Brown

\footnotetext{
Received July 12, 2016.

Accepted October 5, 2016.

${ }^{1}$ Current address: Animal Breeding and Genomics Centre, Wageningen UR Livestock Research, $6700 \mathrm{AH}$ Wageningen, the Netherlands.

${ }^{2}$ Corresponding author: jeremie.vandenplas@wur.nl
}

Swiss sires, included in the InterGenomics consortium at Interbull Centre, into the national evaluation in Slovenia. The results showed that the method could (1) increase reliability of a national (genomic) evaluation; (2) provide consistent ranking of all animals: bulls, cows, and young animals; and (3) increase the size of a genomic training population. These features provide more efficient and transparent selection throughout a breeding program.

Key words: single-step genomic BLUP, combination, multi-national, genomically enhanced estimated breeding values

\section{INTRODUCTION}

This paper presents a national single-step genomic method that integrates multi-national genomic EBV and associated reliabilities. Breeding programs collect phenotypic and genetic data and distill it in the form of EBV and associated reliabilities by using BLUP (Henderson, 1984). Simultaneous use of all data is a condition to obtain unbiased EBV but this condition is not always fully met. For example, most breeding programs make some use of elite parents imported from other populations. When the imported individuals or their relatives are evaluated as a part of the routine national genetic evaluation, only their national data are commonly used. The inability to include foreign data in national evaluations can lead to bias and lower reliability of EBV. This is particularly the case in small breeding programs that often rely more on importation than do large programs.

In dairy cattle, the issue of incomplete across-country data is traditionally tackled through the multiple across-country evaluation of sires (MACE; Schaeffer, 1994). A unit of information in such evaluations is a sire's EBV and associated reliability. Conducted by Interbull Centre (Uppsala, Sweden), MACE simultaneously regresses all the available national information 
onto a joint pedigree to obtain country-specific rankings of all sires without sharing the "raw" data. Because all sires are evaluated on each country scale, MACE has proved to be a valuable source of "phenotypic" data for genomic evaluations. By using the MACE information and exchanging sire genotypes, the national programs have been able to build large training populations that facilitate accurate genomic predictions (VanRaden et al., 2009; Lund et al., 2010; Jorjani et al., 2012).

The most common approach to use MACE information in genomic evaluation is via the so-called multi-step method. The method involves deregressing the MACE EBV to obtain pseudo-records and regressing these on genomic relationships with implicit or explicit blending of the traditional pedigree-based information (VanRaden, 2008). Although breeding programs perform such evaluations nationally, the evaluations involve a multi-national training population with domestic and foreign information. We refer to such an evaluation as a "multi-national genomic evaluation."

An example of the multi-step method is the genomic evaluation in the Brown Swiss breed operated through the InterGenomics consortium at Interbull Centre (Jorjani et al., 2012). Individual Brown Swiss breeding programs in several countries (Austria, France, Germany, Italy, Slovenia, Switzerland, and the United States) have limited training populations for genomic selection because of the limited size of their respective national populations. To enable accurate genomic evaluation, the programs agreed to combine national genotype data sets, and the phenotypic data (the MACE information) are readily available for all sires on each country scale.

An alternative to the multi-step method is the singlestep method that jointly uses phenotypic, genomic, and pedigree data in one analysis (Legarra et al., 2014). Although the multi-step method is practical, it rests on several assumptions that are not met in all situations (Legarra et al., 2014). For example, the multi-step method assumes that pseudo-records are independent. This can be assumed for pseudo-records with high reliability, as is the case with sires tested on a large number of progeny in large breeding programs. However, independence cannot be assumed for pseudo-records with low reliability, as is the case with sires tested on a smaller number of progeny in small breeding programs. When records are dependent, the analysis should take this into account to avoid double counting of information and consequently overestimating reliability (e.g., Calus et al., 2016). The single-step method, referred here to as the single-step genomic BLUP (ssGBLUP), avoids this because it simultaneously utilizes any phenotypic, genomic, and pedigree data in a single evaluation (Legarra et al., 2014).
However, standard ssGBLUP cannot integrate multi-national information. A breeding program with national evaluation based on ssGBLUP needs a way to integrate multi-national information to use all available sources of information optimally. Such a method would have to consider that the multi-national information might be partially based on national information, which needs to be accounted for to avoid double counting. Specifically, double counting can occur due to double use of data that pertains to an individual animal, as well as to double use of data that is correlated among relatives (Vandenplas and Gengler, 2012; Vandenplas et al., 2014). We will refer to these 2 as double counting contributions from records and double counting contributions from relatives, respectively.

The aim of this study was to develop and demonstrate the potential of a national single-step genomic method that integrates multi-national genomic information and avoids the double counting. The method delivers a national genomic evaluation that uses all the available data in an appropriate manner and presents results on the same scale for bulls, cows, and young animals. These 2 properties enable efficient and transparent selection among all animals.

\section{MATERIALS AND METHODS}

The first part of this section describes theory of (1) the standard ssGBLUP, (2) the integration of multinational ssGBLUP information into a national ssGBLUP, and (3) the required corrections to avoid double counting. The second part describes a demonstration.

\section{Theory}

The Standard ssGBLUP. The standard ssGBLUP can be applied to a univariate linear mixed model:

$$
\mathbf{y}=\mathbf{X b}+\mathbf{Z a}+\mathbf{e}
$$

where $\mathbf{y}$ is the vector of phenotypic records, $\mathbf{b}$ is the vector of fixed effects, $\mathbf{a}$ is the vector of random additive genetic effects, and $\mathbf{e}$ is the vector of residuals. The matrices $\mathbf{X}$ and $\mathbf{Z}$ are incidence matrices linking $\mathbf{y}$ with, respectively, $\mathbf{b}$ and $\mathbf{a}$.

In the context of ssGBLUP, it is assumed that $\mathbf{a} \sim \operatorname{MVN}\left(0, \mathbf{H} \sigma_{a}^{2}\right)$, where $M V N=$ multivariate normal, $\mathbf{H}$ is a combined genomic and pedigree relationship matrix, $\sigma_{a}^{2}$ is the additive genetic variance of the trait; and that $\mathbf{e} \sim \operatorname{MVN}\left(0, \mathbf{I} \sigma_{e}^{2}\right)$, where $\mathbf{I}$ is an identity matrix and $\sigma_{e}^{2}$ is the residual variance (Legarra et al., 2014).

The inverse of $\mathbf{H}$ is 


$$
\mathbf{H}^{-1}=\left[\begin{array}{ll}
\mathbf{H}_{n, n} & \mathbf{H}_{n, g} \\
\mathbf{H}_{g, n} & \mathbf{H}_{g, g}
\end{array}\right]^{-1}=\mathbf{A}^{-1}+\left[\begin{array}{lc}
0 & 0 \\
0 & \left(\alpha \mathbf{G}+(1-\alpha) \times \mathbf{A}_{g, g}^{-1}\right)
\end{array}\right],[2]
$$

where the subscript $n$ refers to nongenotyped animals, the subscript $g$ refers to genotyped animals, $\mathbf{A}=\left[\begin{array}{ll}\mathbf{A}_{n, n} & \mathbf{A}_{n, g} \\ \mathbf{A}_{g, n} & \mathbf{A}_{g, g}\end{array}\right]$ is the pedigree relationship matrix, $\mathbf{G}$ is a genomic relationship matrix, and $\alpha$ is a weight used to account for genetic variation not captured by the marker genotypes (Legarra et al., 2014).

In this study, a genomic relationship matrix $\mathbf{G}$ was constructed following the first method of VanRaden (2008), using current allele frequencies and adjusted to have mean of diagonal elements and off-diagonal elements equal to those of $\mathbf{A}_{g, g}$. Other methods to compute G could be used without loss of generality.

Integration of Multi-National Genomic Information that Is Independent of National Information. Consider a national and multi-national ssGBLUP for the same set of individuals with the same pedigree and genotype data but completely independent phenotypic data. We want to integrate the results of multinational ssGBLUP into the national ssGBLUP. For clarity of equations, we assume that individuals whose multi-national genomic information is to be integrated in the national ssGBLUP have all been genotyped and that all their genotypes are available to the national ssGBLUP. However, the developed equations can be applied to more general cases, such as genotyped and nongenotyped foreign animals associated with foreign information. Following Vandenplas et al. (2014), this integration can be performed by adopting a Bayesian view of the linear mixed model [1] and assuming the following prior:

$$
\mathbf{a} \sim \operatorname{MVN}\left(\hat{\mathbf{a}}^{*}, \mathbf{H}^{*}\right),
$$

where $\hat{\mathbf{a}}^{*}=\left[\begin{array}{c}\hat{\mathbf{a}}_{n}^{*} \\ \hat{\mathbf{a}}_{g}^{*}\end{array}\right]$, with $\hat{\mathbf{a}}_{g}^{*}$ being the vector of multinational genomic EBV (GEBV; obtained from the multi-national ssGBLUP) for the genotyped animals and $\hat{\mathbf{a}}_{n}^{*}=\mathbf{H}_{n, g} \mathbf{H}_{g, g}^{-1} \hat{\mathbf{a}}_{g}^{*}$ being the vector of multi-national GEBV for the nongenotyped animals, and $\mathbf{H}^{*}$ is the matrix of prediction error covariances of $\hat{\mathbf{a}}^{*}$.

The inverse of $\mathbf{H}^{*}$ is

$$
\mathbf{H}^{*-1}=\mathbf{H}^{-1} \sigma_{a}^{-2}+\boldsymbol{\Lambda}^{*}=\mathbf{H}^{-1} \sigma_{a}^{-2}+\left[\begin{array}{cc}
0 & 0 \\
0 & \boldsymbol{\Lambda}_{g, g}^{*}
\end{array}\right],
$$

where the matrix $\boldsymbol{\Lambda}_{g, q}^{*}$ is a diagonal variance matrix with the ith element equal to $E R C_{i}^{*} \sigma_{e}^{-2}$ and $E R C_{i}^{*}$ being the effective number of records (Misztal and Wiggans, 1988; also called "effective record contributions") that contributed to the multi-national ssGBLUP for the $i$ th animal (Vandenplas et al., 2014). Because contributions from relationships are already accounted through the matrix $\mathbf{H}^{-1}$, the effective number of records must be free of contributions from relatives (Vandenplas and Gengler, 2012).

Estimation of the Effective Number of Records Free of Contributions from Relatives. In the context of traditional pedigree-based genetic evaluation, Vandenplas and Gengler (2012) developed an algorithm (named TSA) to estimate $E R C_{i}^{*}$ in 2 steps. We only provide a summary of the algorithm here for completeness. The first step identifies animals in the multi-national evaluation associated with $E R C_{i}^{*}$ equal to zero. This identification involves the estimation of a matrix D such that the diagonal elements of the matrix $\mathbf{P}^{-1}=\left(\mathbf{D} \sigma_{e}^{-2}+\mathbf{A}_{g, g}^{-1} \sigma_{a}^{-2}\right)^{-1}$ are sufficiently close to the prediction error variances of the multi-national evaluation. The matrix $\mathbf{P}$ mimics the part of the left-handside of system of equations for the additive genetic effect of the multi-national evaluation. The diagonal elements of $\mathbf{D}$ approximate $E R C_{i}^{*}$. Knowing animals associated with $E R C_{i}^{*}$ equal to zero, the second step computes $E R C_{i}^{*}$, similarly to the first step, but with a matrix $\mathbf{P}$ that mimics the full system of equations of the multi-national evaluation. This can be done by absorbing one hypothetical fixed effect that takes into account that some animals may have $E R C_{i}^{*}$ equal to zero, and that mimics the unknown fixed effects considered in the multi-national evaluation.

In our context, modifications of the algorithm of Vandenplas and Gengler (2012) are required because both the national and multi-national ssGBLUP use both pedigree and genomic relationships. Therefore, in addition to the contributions from relatives via pedigree relationships, the contributions from relatives via genomic relationships must be taken into account. Contributions from both sources can be taken into account by replacing $\mathbf{A}_{g, g}^{-1}$ with $\mathbf{H}_{g, g}^{-1}$.

Integration of Multi-National Genomic Information that Is Not Independent of National Information. Consider a national and multi-national ssGBLUP for the same set of individuals with the same pedigree and genotype data and partially dependent phenotypic data. This situation can arise through exchange of data between breeding programs or, more commonly, by using the MACE information as pheno- 
types, which combines information from several national evaluations. In such a situation, the integration of multi-national information into the national ssGBLUP must avoid double counting of contributions that arises from the partially dependent phenotypic data (i.e., double counting of contributions from records). This latter double counting must be avoided in addition to double counting of contributions from relatives. Consideration of the double counting of contributions from records can be achieved by using a hypothetical pedigree-based evaluation, which is run for all individuals using the partially dependent phenotypic data, summarized by the vector $\mathbf{y}^{\#}$. The system of equations for this hypothetical pedigree-based evaluation can be written as

$$
\left(\mathbf{A}^{-1} \sigma_{a}^{-2}+\Lambda^{* *}\right) \hat{\mathbf{a}}^{\#}=\Lambda^{* *} \mathbf{y}^{\#}
$$

where $\hat{\mathbf{a}}^{\#}$ is the vector of $\mathrm{EBV}$, and the matrix $\Lambda^{* *}=\left[\begin{array}{cc}0 & 0 \\ 0 & \Lambda_{g, g}^{* *}\end{array}\right]$, with $\boldsymbol{\Lambda}_{g, g}^{* *}$ being a diagonal variance matrix with the $i$ th element equal to $E R C_{i}^{* *} \sigma_{e}^{-2}$, and $E R C_{i}^{* *}$ is the effective number of records of the $i$ th animal that contributed to the hypothetical pedigree-based evaluation. Values for $E R C_{i}^{* *}$ are computed as described before, but this time with the original algorithm of Vandenplas and Gengler (2012). This evaluation is hypothetical because the vector $\mathbf{y}^{\#}$ of partially dependent phenotypic data is unknown.

Multi-national genomic information, dependent of national information, can be integrated into the national ssGBLUP by assuming the following prior (see Vandenplas et al. (2014) for details):

$$
\mathbf{a} \sim \operatorname{MVN}\left(\hat{\mathbf{a}}^{* *}, \mathbf{H}^{* *}\right),
$$

where the vector $\hat{\mathbf{a}}^{* *}$ is

$$
\hat{\mathbf{a}}^{* *}=\mathbf{H}^{* *}\left[\mathbf{H}^{*-1} \hat{\mathbf{a}}^{*}-\left(\mathbf{A}^{-1} \sigma_{a}^{-2}+\boldsymbol{\Lambda}^{* *}\right) \hat{\mathbf{a}}^{\#}\right],
$$

and the inverse of $\mathbf{H}^{* *}$ is

$$
\mathbf{H}^{* *-1}=\mathbf{H}^{*-1}-\mathbf{\Lambda}^{* *}=\mathbf{H}^{*-1}-\left[\begin{array}{cc}
0 & 0 \\
0 & \Lambda_{g, g}^{* *}
\end{array}\right] .
$$

The vector $\hat{\mathbf{a}}^{* *}$ contains GEBV of a (hypothetical) multi-national ssGBLUP independent of national information, and the matrix $\mathbf{H}^{* *}$ is the prediction error (co) variance matrix associated with $\hat{\mathbf{a}}^{* * *}$,
The system of equations of the national ssGBLUP that integrates the dependent multi-national genomic information and avoids double counting, is

$$
\left[\begin{array}{cc}
\mathbf{X}^{\prime} \mathbf{R}^{-1} \mathbf{X} & \mathbf{X}^{\prime} \mathbf{R}^{-1} \mathbf{Z} \\
\mathbf{Z}^{\prime} \mathbf{R}^{-1} \mathbf{X} & \mathbf{Z}^{\prime} \mathbf{R}^{-1} \mathbf{Z}+\mathbf{H}^{* *-1}
\end{array}\right]\left[\begin{array}{l}
\hat{\mathbf{b}} \\
\hat{\mathbf{a}}
\end{array}\right]=\left[\begin{array}{l}
\mathbf{X}^{\prime} \mathbf{R}^{-1} \mathbf{y} \\
\mathbf{Z}^{\prime} \mathbf{R}^{-1} \mathbf{y}+\mathbf{H}^{* *-1} \hat{\mathbf{a}}^{* *}
\end{array}\right],[8]
$$

where $\mathbf{R}$ is the residual (co)variance matrix. Fortunately, the vector $\hat{\mathbf{a}}^{* *}$ in the equation [8] need not be computed explicitly. Using equations [5-7], the lefthand side of [8] simplifies to

$$
\left[\begin{array}{cc}
\mathbf{X}^{\prime} \mathbf{R}^{-1} \mathbf{X} & \mathbf{X}^{\prime} \mathbf{R}^{-1} \mathbf{Z} \\
\mathbf{Z}^{\prime} \mathbf{R}^{-1} \mathbf{X} & \mathbf{Z}^{\prime} \mathbf{R}^{-1} \mathbf{Z}+\mathbf{H}^{-1} \sigma_{a}^{-2}+\mathbf{\Lambda}^{*}-\boldsymbol{\Lambda}^{* *}
\end{array}\right]
$$

and the right-hand side of [8] simplifies to

$$
\left[\begin{array}{l}
\mathbf{X}^{\prime} \mathbf{R}^{-1} \mathbf{y} \\
\mathbf{Z}^{\prime} \mathbf{R}^{-1} \mathbf{y}+\left(\mathbf{H}^{-1} \sigma_{a}^{-2}+\mathbf{\Lambda}^{*}\right) \hat{\mathbf{a}}^{*}-\left(\mathbf{A}^{-1} \sigma_{a}^{-2}+\boldsymbol{\Lambda}^{* *}\right) \hat{\mathbf{a}}^{\#}
\end{array}\right] \cdot[10]
$$

We refer to this type of evaluation as a national ssGBLUP with integration, because it uses national data $(\mathbf{y})$ and integrates multi-national genomic information $\left(\hat{\mathbf{a}}^{*}\right.$ and $\left.\boldsymbol{\Lambda}^{*}\right)$ through prior distribution for breeding values. The double counting is avoided by using $\hat{\mathbf{a}}^{\#}$ and $\boldsymbol{\Lambda}^{* * *}$, which corrects for the partial dependencies.

\section{Demonstration}

The demonstration considered the integration of multi-national genomic information (InterGenomics; Jorjani et al., 2012) into the national evaluation of Brown-Swiss dairy cattle in Slovenia (Potočnik et al., 2000). Figure 1 depicts the flow of data between national and multi-national (genomic) evaluations for the Slovenian situation. The motivation behind this demonstration was to have an evaluation that fulfills 4 criteria:

(1) Use all the available information jointly.

(2) Deliver combined ranking of all animals: bulls, cows, and young animals.

(3) Integrate multi-national genomic information into national evaluation to propagate foreign information onto domestic animals that did not participate in the multi-national genomic evaluation.

(4) Avoid double counting, because the multi-national genomic information is dependent on the national information through MACE. 


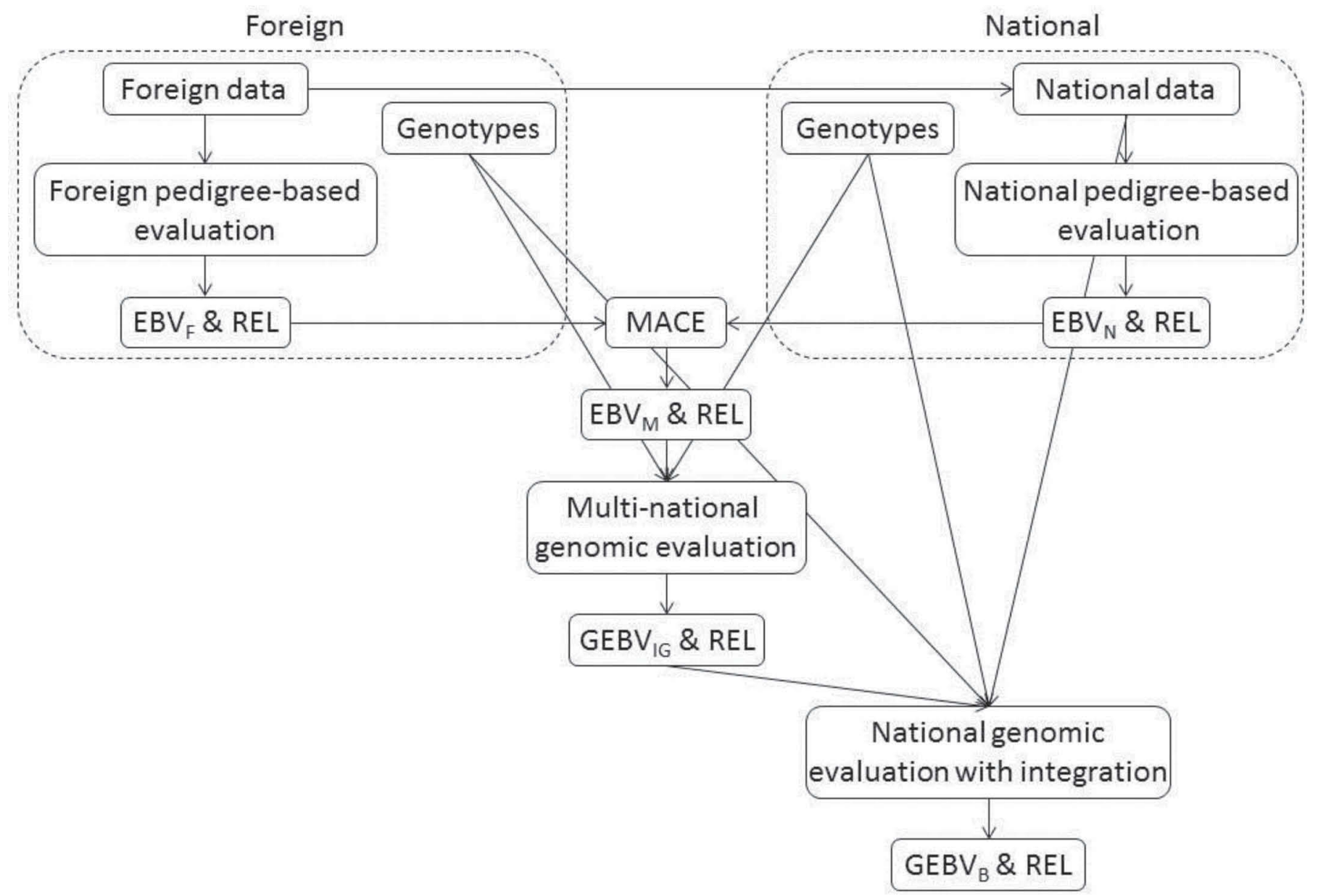

Figure 1. Flow of data between national and multi-national genomic (evaluations) for the Slovenian situation. MACE $=$ multiple acrosscountry evaluation; $\mathrm{REL}=$ reliability; $\mathrm{GEBV}=$ genomically enhanced $\mathrm{EBV} ; \mathrm{EBV}_{\mathrm{F}}=\mathrm{EBV}$ obtained from a foreign pedigree-based evaluation; $\mathrm{EBV}_{\mathrm{M}}=\mathrm{EBV}$ obtained from MACE; $\mathrm{EBV}_{\mathrm{N}}=\mathrm{EBV}$ obtained from the national pedigree-based evaluation; GEBV $\mathrm{IG}_{\mathrm{I}}=\mathrm{GEBV}_{\mathrm{obtained}}$ from the multi-national genomic evaluation; and $\mathrm{GEBV}_{\mathrm{B}}=\mathrm{GEBV}$ obtained from the national genomic evaluation with integration.

The proposed method fulfills all the criteria. In addition, as a by-product, it upgrades national pedigreebased evaluation into a genomic evaluation, because the integration also uses the multi-national genomic relationship matrix.

The different evaluations and notation for the associated EBV were (1) national pedigree-based evaluation $\left(\mathbf{E B V}_{\mathbf{N}}, \hat{\mathbf{a}}^{\#}\right.$ in [9]); (2) multi-national genomic evaluation $\left(\mathbf{G E B V}_{\mathbf{I G}}, \hat{\mathbf{a}}^{*}\right.$ in $\left.[9]\right)$; and (3) national genomic evaluation with integration $\left(\mathbf{G E B V}_{\mathbf{B}}\right.$, $\hat{\mathbf{a}}$ in [8]). National data comprised 1,286,698 test-day records for milk, fat, and protein yields for 56,764 cows in Slovenia, recorded between the years 2000 and 2014. The associated pedigree comprised 101,522 animals.

Multi-national genomic information was obtained from the second routine InterGenomics evaluation in 2014 for 5,852 bulls, and included the GEBV $_{\text {IG }}$ and associated reliabilities. Although the InterGenomics evaluation is based on a multi-step method, we assumed that it is equivalent to a single-step method.

Genotypes were available through the InterGenomics consortium. After editing, genotypes for 5,852 bulls at 38,863 markers were used. Only 191 bulls were genotyped in Slovenia. Edits were performed to avoid possible genotyping errors and misidentifications. The edits involved (1) removal of markers that had minor allele frequency $<0.05$ or genotype call rate $<0.90,(2)$ removal of animals that had genotype call rate $<0.90$, and (3) removal of animals if these animals had Mendelian conflicts with their sire at more than $2 \%$ of markers.

Computations were performed using the BLUPF90 program (Misztal, 2013) modified to include the developed method. National data were used for both the national pedigree-based evaluation and the national genomic evaluation with integration. The national 
pedigree-based evaluation uses a single-trait multiplelactation repeatability model (Potočnik et al., 2000). The national genomic evaluation with integration included genotypes for 5,852 bulls, as well as $\mathrm{GEBV}_{\mathrm{IG}}$ and associated reliabilities for these bulls comprising their multi-national genomic information.

The double counting was avoided in 2 ways. First, we avoided double counting of contributions from records by determining which bull's national information (i.e., its $\mathrm{EBV}_{\mathrm{N}}$ and associated reliability) contributed to MACE, and therefore also to the multi-national genomic evaluation. This determination is based on the nonzero national effective daughter contributions reported by Interbull Centre. Among the 5,852 bulls with the multi-national genomic information, 277 bulls had nonzero national effective daughter contributions, meaning that these 277 bulls' national information contributed to MACE. The $\mathrm{EBV}_{\mathrm{N}}$ and associated reliabilities for these bulls summarized the partial dependence and were used to avoid double counting of contributions from records. Second, we avoided double counting of contributions from relatives with the TSA algorithm, for both the national information and multi-national genomic information. The GEBV $\mathrm{IG}_{\mathrm{IG}}$ were harmonized by adjusting the scale and mean difference toward $\mathrm{EBV}_{\mathrm{N}}$. The weight $\alpha[2]$ was set to 0.85 for milk yield and 0.95 for fat and protein yields. These weights provided the best model fit associated with a national ssGBLUP without integration, as measured by trial and error and compared using the Akaike information criteria.

Reliability (REL) of the national pedigree-based evaluation and national genomic evaluation with integration for the $i$ th animal was computed as $\mathrm{REL}_{i}=1-\mathrm{PEV}_{i} \sigma_{\mathrm{a}}^{-2}$ (ignoring inbreeding), where $\mathrm{PEV}_{i}$ is the prediction error variance obtained from the inverse of the left-hand side of the system of equations.

Analysis of evaluations was performed in 2 ways. First, we compared the national genomic evaluation with integration to the multi-national genomic evaluation. The multi-national genomic evaluation was considered as the reference, because it uses all the national and foreign information. If integration was successful, we should detect no differences in results for animals that participated in both evaluations. The comparison was performed separately for (a) 319 bulls with phenotyped daughters in the national data set, and (b) 5,533 bulls without phenotyped daughters in the national data set. The 319 bulls had, on average, 135 phenotyped daughters in the national data set. The comparison was based on Spearman rank correlations between the different types of EBV, regression of $\mathrm{GEBV}_{\mathrm{IG}}$ on $\mathrm{EBV}_{\mathrm{N}}$, regression of $\mathrm{GEBV}_{\mathrm{IG}}$ on $\mathrm{GEBV}_{\mathrm{B}}$, and reliabilities of $(\mathrm{G}) \mathrm{EBV}$. Additionally, regressions of reliabilities of $\mathrm{GEBV}_{\mathrm{IG}}$ on reliabilities of $\mathrm{GEBV}_{\mathrm{B}}$ were performed to evaluate the effects of double-counting contributions from relatives or from records. Second, we compared national genomic evaluation with integration to the national pedigree-based evaluation to evaluate the gain brought about by integration. The comparison was performed separately for (a) the 43,007 domestic cows that had their own phenotype records in the national data set, (b) the 23 imported cows that had their own phenotype records in the national data set, and (c) the 1,971 domestic bulls and cows that did not have their own phenotype records in the national data set. All animals considered in the comparison were sired by bulls evaluated in the InterGenomics consortium. The comparison was based on Spearman rank correlations between $\mathrm{EBV}_{\mathrm{N}}$ and $\mathrm{GEBV}_{\mathrm{B}}$, and reliabilities of $(\mathrm{G})$ EBV.

\section{RESULTS}

The results showed that the developed method integrated multi-national genotypes, GEBV, and reliabilities into a national evaluation with almost no double counting for bulls with multi-national information. This information was propagated to relatives as shown by the increased reliabilities for the animals that had little information in the national data set.

\section{Demonstration of the National Genomic Evaluation with Integration}

The developed method enabled integration of the multi-national information into the national evaluation for bulls with multi-national information. This is shown in Tables 1 and 2, which compare the national pedigreebased evaluation without integration and the national genomic evaluation with integration to the multi-national genomic evaluation. The multi-national genomic evaluation was considered as a reference. This means that a high correlation and a regression coefficient close to 1.00 was sought between the multi-national genomic evaluation and national genomic evaluation with integration. A total of 319 (national or foreign) bulls had at least one phenotyped daughter in the national data set. This group included the 277 bulls whose national information contributed to MACE. For the 319 bulls, rank correlations between the national pedigree-based evaluation without integration and the multi-national genomic evaluation ranged from 0.76 to 0.79 for the 3 traits, whereas integration increased these correlations to $\geq 0.99$ (Table 1). Integration also improved regressions of the national pedigree-based evaluation onto the multi-national genomic evaluation (the regression coefficient was closer to 1.00) and increased aver- 
Table 1. Comparison of the national pedigree-based evaluation without integration $(\mathrm{N})$ and national genomic evaluation with integration (B) to the multi-national genomic evaluation (IG) for the 319 bulls with phenotyped daughters in the national data set ${ }^{1}$

\begin{tabular}{llccll}
\hline Trait & Evaluation & $\mathrm{r}$ & $\mathrm{b}$ & $\mathrm{R}^{2}$ & Reliability \\
\hline Milk yield & $\mathrm{N}$ & 0.79 & $0.92(0.040)$ & 0.68 & $0.87(0.19)$ \\
& $\mathrm{B}$ & $>0.99$ & $1.00(0.003)$ & 0.99 & $0.97(0.02)$ \\
Fat yield & $\mathrm{IG}$ & - & - & - & $0.97(0.02)$ \\
& $\mathrm{N}$ & 0.76 & $0.90(0.040)$ & 0.62 & $0.87(0.20)$ \\
Protein yield & $\mathrm{B}$ & 0.99 & $1.01(0.003)$ & 0.99 & $0.97(0.02)$ \\
& $\mathrm{IG}$ & - & - & - & $0.97(0.02)$ \\
& $\mathrm{N}$ & 0.76 & $0.90(0.040)$ & 0.62 & $0.87(0.19)$ \\
& $\mathrm{B}$ & 0.99 & $1.00(0.003)$ & 0.99 & $0.97(0.02)$ \\
& $\mathrm{IG}$ & - & - & - & $0.97(0.02)$ \\
\hline
\end{tabular}

${ }^{1} \mathrm{r}=$ rank correlation between genomic $(\mathrm{G}) \mathrm{EBV}_{\mathrm{IG}}$ and $\mathrm{EBV}_{\mathrm{N}}$ and between $\mathrm{GEBV}_{\mathrm{IG}}$ and $\mathrm{GEBV}_{\mathrm{B}} ; \mathrm{b}=$ regression coefficient ( $\mathrm{SE}$ in parentheses) and $\mathrm{R}^{2}=$ associated coefficient of determination (regression of $\mathrm{GEBV}_{\mathrm{IG}}$ on $\mathrm{EBV}_{\mathrm{N}}$ and of $\mathrm{GEBV}_{\mathrm{IG}}$ on $\mathrm{GEBV}_{\mathrm{B}}$ ); reliability = average reliability (SD in parentheses).

age reliability from 0.87 to 0.97 (Table 1 ). The same was observed for the 5,533 bulls without phenotyped daughters in the national data set. The rank correlations between the national pedigree-based evaluation without integration and the multi-national genomic evaluation for these bulls were in the range from 0.51 to 0.60 for the 3 traits, and integration increased these correlations above 0.99 (Table 2). Reliability for these bulls increased from 0.17 to 0.90 and above (Table 2).

The developed method avoided most double counting when the multi-national information was integrated into the national evaluation. This is shown for the milk yield trait in Tables 1-3, and Figures 2 and 3 . Double counting of contributions from relatives or records biased $\mathrm{GEBV}_{\mathrm{B}}$, as shown by coefficients of the regression of $\mathrm{GEBV}_{\mathrm{IG}}$ on $\mathrm{GEBV}_{\mathrm{B}}<1$ when double counting was not avoided (Table 3). Double counting also led to higher average reliabilities for the national genomic evaluation with integration than those for the multi-national genomic evaluation (Tables 1-3). Higher reliabilities with the national genomic evaluation with integration than with the multi-national genomic evaluation could indicate double counting, because both evaluations used the same information and therefore should not have different reliabilities. Reliabilities were, on average, equivalent between the multi-national genomic evaluation and the national genomic evaluation with integration (Tables 1-3), which indicates that the developed method avoided almost all double counting.

Effect of potential double counting of contributions from relatives was minimal in the demonstration. This is shown in Table 3, which demonstrates the national genomic evaluation with integration when we avoided double counting of contributions from both records and relatives or only from records. Only results for milk yield are shown. The correlation was $>0.99$ and did not differ when we avoided double counting or not, but the regression coefficient improved from 0.98 or 0.99 to 1.00 when we avoided double counting. When we did not avoid double counting, the reliabilities were higher, especially for the lowest values (Table 3; Figures 2 and 3 ), because the evaluation was not informed that the same information was used twice. However, on average, the overestimation of reliability in the demonstration was minimal, at most 0.01 (Table 3 ), because they were already close to the upper bound of 1 .

Table 2. Comparison of the national pedigree-based evaluation without integration $(\mathrm{N})$ and national genomic evaluation with integration (B) to the multi-national genomic evaluation (IG) for the 5,533 bulls without phenotyped daughters in the national data set $^{1}$

\begin{tabular}{llcccc}
\hline Trait & Evaluation & $\mathrm{r}$ & $\mathrm{b}$ & $\mathrm{R}^{2}$ & Reliability \\
\hline Milk yield & $\mathrm{N}$ & 0.55 & $1.40(0.030)$ & 0.35 & $0.17(0.10)$ \\
& $\mathrm{B}$ & $>0.99$ & $1.00(0.000)$ & $>0.99$ & $0.91(0.02)$ \\
Fat yield & $\mathrm{IG}$ & - & - & - & $0.90(0.02)$ \\
& $\mathrm{N}$ & 0.51 & $1.41(0.030)$ & 0.33 & $0.17(0.10)$ \\
Protein yield & $\mathrm{B}$ & $>0.99$ & $1.00(0.000)$ & $>0.99$ & $0.90(0.02)$ \\
& $\mathrm{IG}$ & - & - & - & $0.90(0.02)$ \\
& $\mathrm{N}$ & 0.60 & $1.53(0.020)$ & 0.41 & $0.17(0.10)$ \\
& $\mathrm{B}$ & $>0.99$ & $1.00(0.000)$ & $>0.99$ & $0.90(0.02)$ \\
& $\mathrm{IG}$ & - & - & - & $0.90(0.02)$ \\
\hline
\end{tabular}

${ }^{1} \mathrm{r}=$ rank correlation between genomic $(\mathrm{G}) \mathrm{EBV}_{\mathrm{IG}}$ and $\mathrm{EBV}_{\mathrm{N}}$ and between $\mathrm{GEBV}_{\mathrm{IG}}$ and $\mathrm{GEBV}_{\mathrm{B}}$; $\mathrm{b}=$ regression coefficient ( $\mathrm{SE}$ in parentheses) and $\mathrm{R}^{2}=$ associated coefficient of determination (regression of $\mathrm{GEBV}_{\mathrm{IG}}$ on $\mathrm{EBV}_{\mathrm{N}}$ and of $\mathrm{GEBV}_{\mathrm{IG}}$ on $\mathrm{GEBV}_{\mathrm{B}}$ ); reliability = average reliability (SD in parentheses). 
Table 3. Comparison of the milk yield national genomic evaluation with integration (B) to the multi-national genomic evaluation (IG) when double counting contributions from relatives or from records was avoided or not ${ }^{1}$

\begin{tabular}{|c|c|c|c|c|c|}
\hline Double counting & $\mathrm{r}$ & $\mathrm{b}$ & $\mathrm{R}^{2}$ & Reliability & $b_{\text {REL }}$ \\
\hline \multicolumn{6}{|c|}{$\begin{array}{l}\text { Bulls with phenotyped daughters in the national data } \\
\text { set }(\mathrm{n}=319)\end{array}$} \\
\hline Both avoided & $>0.99$ & $1.00(0.003)$ & 0.99 & $0.97(0.02)$ & $1.06(0.01)$ \\
\hline \multicolumn{6}{|c|}{$\begin{array}{l}\text { Bulls without phenotyped daughters in the national } \\
\text { data set }(\mathrm{n}=5,533)\end{array}$} \\
\hline Both avoided & $>0.99$ & $1.00(0.000)$ & $>0.99$ & $0.91(0.02)$ & $1.02(0.001)$ \\
\hline
\end{tabular}

${ }^{1} \mathrm{r}=$ rank correlation between genomic $(\mathrm{G}) \mathrm{EBV}_{\mathrm{IG}}$ and $\mathrm{GEBV}_{\mathrm{B}} ; \mathrm{b}=$ regression coefficient (SE in parentheses) and $\mathrm{R}^{2}=$ associated coefficient of determination (regression of $\mathrm{GEBV}_{\mathrm{IG}}$ on $\mathrm{GEBV}_{\mathrm{B}}$ ); reliability = average reliability (SD in parentheses); $\mathrm{b}_{\mathrm{REL}}=\mathrm{regression}_{\mathrm{s}}$ coefficient $(\mathrm{SE}$ in parentheses) for regression of reliabilities of $\mathrm{GEBV}_{\mathrm{IG}}$ on reliabilities of $\mathrm{GEBV}_{\mathrm{B}}$.

Effect of potential double counting of contributions from records was also minimal in our demonstration and mainly affected the evaluation of the 319 bulls with phenotyped daughters in the national data set. This is shown in Table 3, which demonstrates the national genomic evaluation with integration when we avoided double counting of contributions from both relatives and records or only from relatives. The correlation did not differ when we avoided double counting or not, but the regression coefficient improved from 0.99 to 1.00 when we avoided double counting (Table 3). Higher reliabilities were also observed on average for the bulls with phenotyped daughters in the national data set when we did not avoid double counting of contributions from records. Avoiding this type of double counting led to reliabilities of the national genomic evaluation with integration similar to the reliabilities of the multi-national genomic evaluation (Tables 1-3; Figures 2 and 3). Higher reliabilities for some bulls are likely due to various approximations and computations, for example, of (multi-)national reliabilities and $E R C_{i}^{* *}$.

\section{Comparison of the National Evaluation Without and With Integration}

Integration of the multi-national genomic information into the national evaluation increased reliability for animals with low amounts of information in the national data set. This is shown in Table 4 and Figure 4. Table 4 compares the national pedigree-based evaluation without integration and the national genomic evaluation with integration. For the domestic cows with phenotypes in the national data set, the reliability did not increase with integration, and rank correlation between the 2 evaluations was $>0.99$ for all 3 traits. For the imported cows with phenotypes in the national data set, the reliability increased slightly, from 0.64 for fat yield and from 0.67 to 0.70 for milk and protein yields. The integration had little effect on ranking of these cows. Figure 4 compares reliabilities from the national genomic evaluation with integration to reliabilities from the national pedigree-based evaluation without integration for animals without own phenotype in the national data set. Figure 4 shows that there was no change in reliability for animals that already had high reliability before integration (progeny-tested bulls), whereas reliability increased for animals that had low reliability before integration (nonphenotyped animals). For example, animals with reliability of $<0.25$ before integration received, with integration, an average increase in reliability of 0.08 .

\section{DISCUSSION}

Our results highlight 3 main points for discussion: (1) the developed method, (2) implications for breeding programs using the developed method, and (3) other potential applications of the developed method.

\section{The Developed Method}

The results showed that the developed method was successful in integrating multi-national genomic information into a national evaluation and achieved this without double counting the national information. To our knowledge, this is the first time that the results of a multi-national genomic-based evaluation have been integrated into a national genomic evaluation. This work builds on previous theoretical and applied work (Quaas and Zhang, 2006; Legarra et al., 2007; Vandenplas and Gengler, 2012; Vandenplas et al., 2013), in particular on the previous work of integrating multi-national pedigree-based (MACE) information into a national pedigree-based evaluation (Vandenplas et al., 2014) or 


\section{Both avoided}

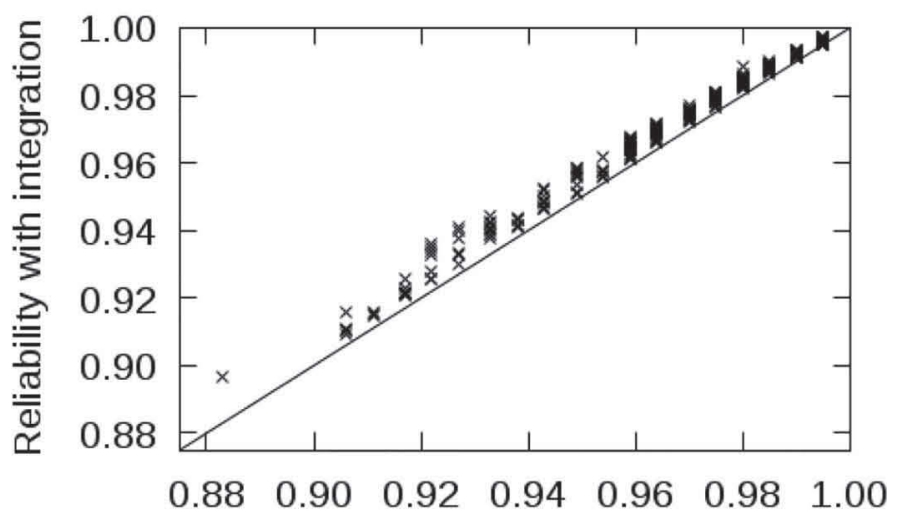

Relatives - Not avoided

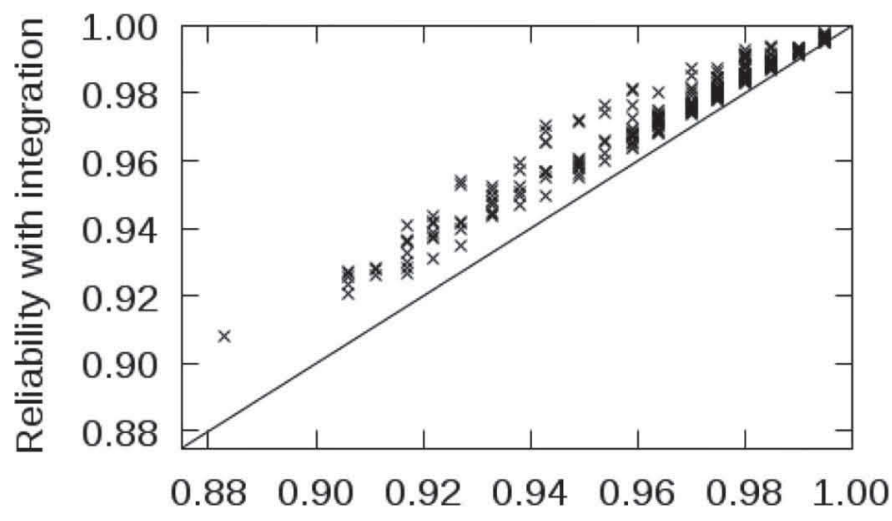

Records - Not avoided

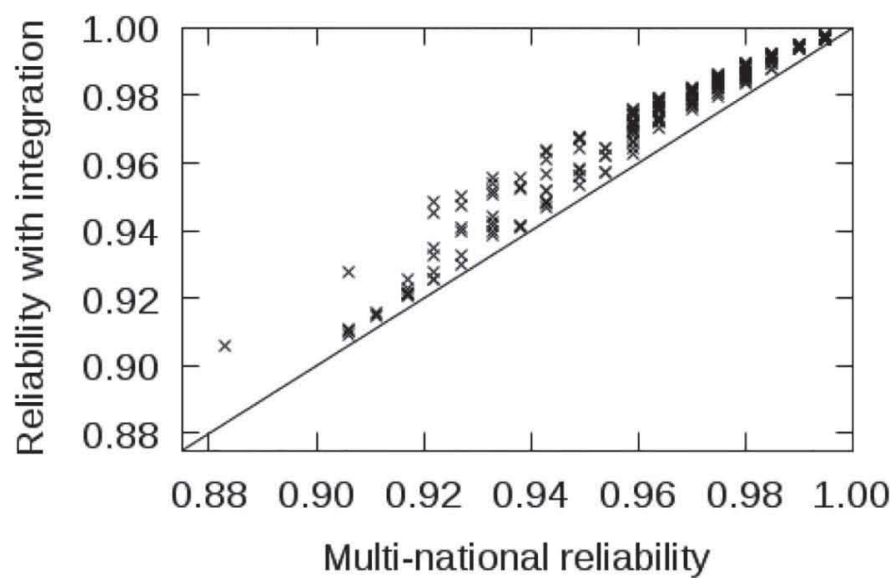

Figure 2. Reliabilities from the national genomic evaluation with integration against reliabilities from the multi-national genomic evaluation for milk yield in bulls with phenotyped daughters in the national data set. Double counting of contributions from relatives (records) was avoided or not.
Both avoided

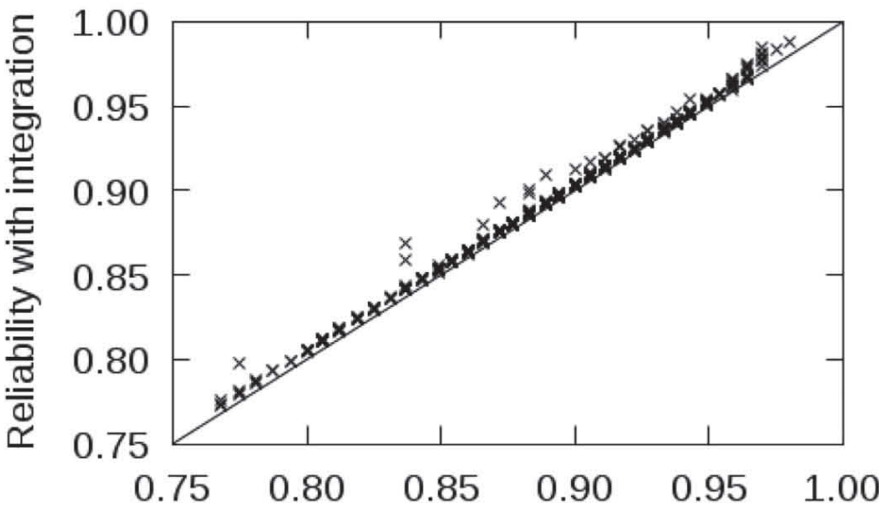

Relatives - Not avoided

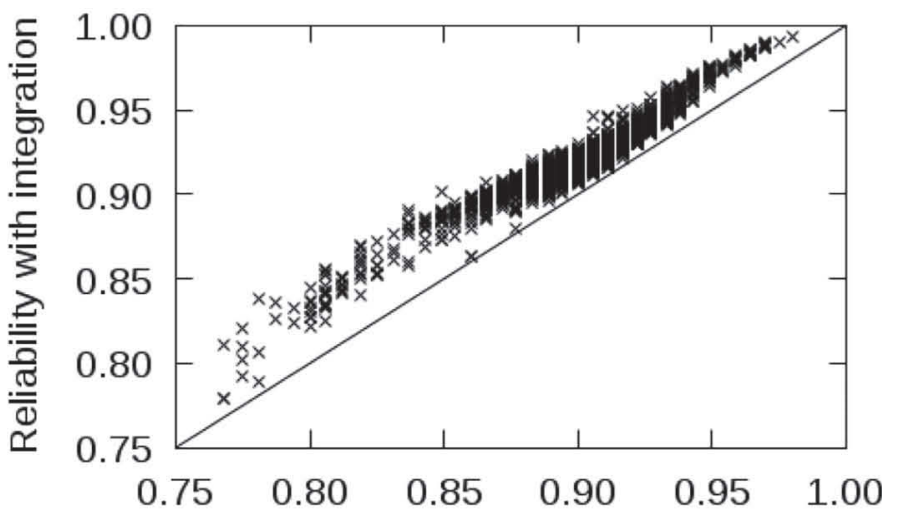

Records - Not avoided

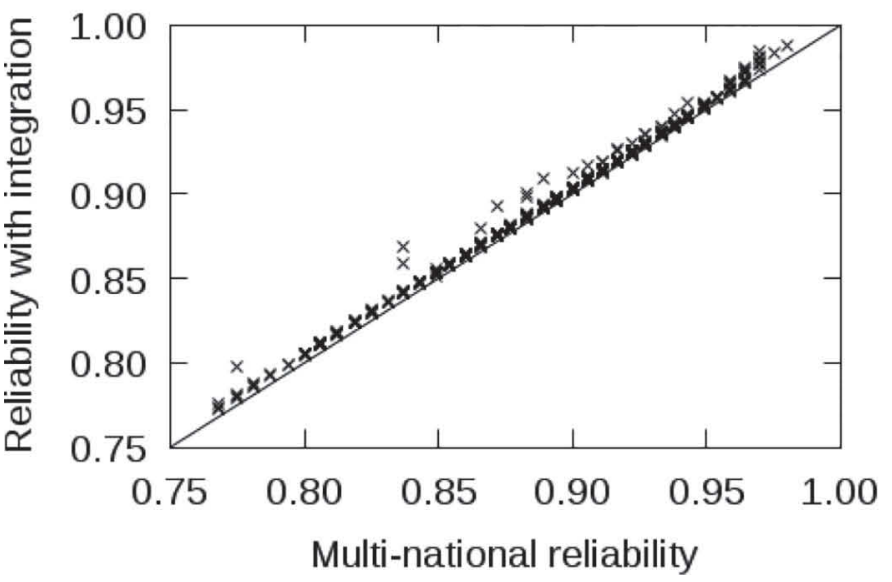

Figure 3. Reliabilities from the national genomic evaluation with integration against reliabilities from the multi-national genomic evaluation for milk yield in bulls without phenotyped daughters in the national data set. Double counting of contributions from relatives (records) was avoided or not. 


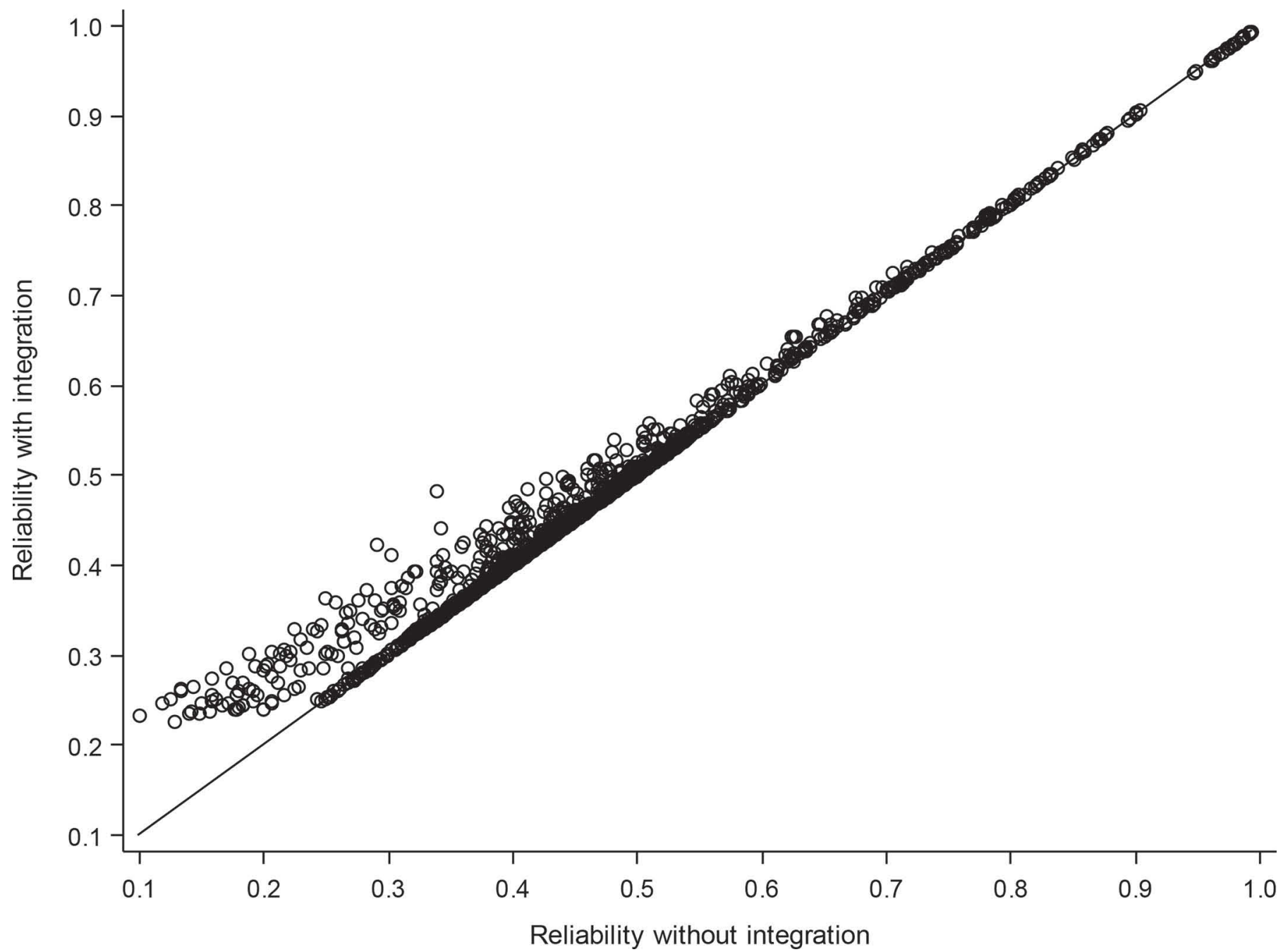

Figure 4. Reliabilities from the national genomic evaluation with integration against reliabilities from the national pedigree-based evaluation without integration for milk yield in domestic animals (bulls and cows) without own phenotype in the national data set.

a national genomic evaluation (Colinet et al., 2013). The demonstration of the method gave a national genomic evaluation with integration, which compared well with the multi-national genomic evaluation, both for the bulls that had phenotyped daughters in the national data set and for those that did not. We based

Table 4. Comparison of the national pedigree-based evaluation without integration $(\mathrm{N})$ and the national genomic evaluation with integration $(B)^{1}$

\begin{tabular}{lccc}
\hline Trait & $\mathrm{r}$ & Reliability $_{\mathrm{N}}$ & Reliability $_{\mathrm{B}}$ \\
\hline $\begin{array}{l}\text { Domestic cows with own phenotype records in the } \\
\text { national data set }(\mathrm{n}=43,007)\end{array}$ & & & \\
$\quad$ Milk yield & $>0.99$ & $0.74(0.09)$ & $0.74(0.09)$ \\
$\quad$ Fat yield & $>0.99$ & $0.71(0.09)$ & $0.72(0.09)$ \\
$\quad$ Protein yield & & $0.74(0.09)$ & $0.74(0.09)$ \\
Imported cows with own phenotype records in the & & \\
national data set $(\mathrm{n}=23)$ & 0.98 & $0.67(0.14)$ & $0.70(0.12)$ \\
$\quad$ Milk yield & 0.98 & $0.64(0.16)$ & $0.67(0.13)$ \\
Fat yield & 0.99 & $0.67(0.15)$ & $0.70(0.12)$ \\
Protein yield & & &
\end{tabular}


the method on the ssGBLUP framework (Legarra et al., 2014), which naturally uses all phenotype, pedigree, and genotype data, and now also the results of an external genetic (genomic) evaluation. The method assumes that ssGBLUP is used both at the national and multi-national level. The latter was not the case in the demonstration, but the InterGenomics' evaluation through the multi-step method using a genomic relationship matrix and blending with the pedigree-based evaluation can be seen as approximately equivalent to ssGBLUP (VanRaden et al., 2009; Aguilar et al., 2010).

The developed method emphasizes the importance of avoiding double counting of dependent sources of information. Any genetic evaluation aggregates the animal's own phenotypes and those of relatives through a relationship matrix (Misztal and Wiggans, 1988). An evaluation that attempts to integrate EBVs from some other evaluation needs to account for this aggregation to avoid counting the same contributions more than once. This is of particular importance for the setting as used in this study, because the multi-national genomic EBV are commonly a result of 3 consecutive evaluations that accumulate information from each of the previous ones. The first is the national pedigree-based evaluation; the second is the multi-national pedigree-based evaluation (MACE); and the third is the multi-national genomic evaluation, which can be performed truly multi-nationally as in the case of InterGenomics (Jorjani et al., 2012) or nationally but using multi-national data (e.g., Lund et al., 2010; Prribyl et al., 2013). Integrating the results of the third into the first therefore must avoid counting national information twice. Although this is commonly taken into account for an animal's own contributions in the context of multi-national pedigree-based evaluations (e.g., Prribyl et al., 2013; VanRaden et al., 2014), it is less often taken into account for contributions from relatives (Calus et al., 2016). The developed method takes this into account.

The potential of double counting of contributions from relatives or from records in the demonstration was limited. For example, when double counting of contributions from relatives was not avoided, the reliability of EBVs was overestimated by only 0.01 and the slope of regression was just below 1.00 (Table 3). When this type of double counting was avoided, the reliability was as expected and the slope of regression of $\mathrm{GEBV}_{\mathrm{IG}}$ on $\mathrm{GEBV}_{\mathrm{B}}$ was 1.00. These results show the phenomenon of double counting (reliability is overestimated when double counting is not avoided) and that the method works (reliability is not overestimated when double counting is avoided). However, in this demonstration, avoiding the double counting of contributions from relatives did not have much effect, especially on GE$\mathrm{BV}_{\mathrm{B}}$. This is expected, because most bulls had highly reliable GEBV, which means that their GEBV have high contribution of their "own" effective records and less so on relatives' records (Sigurdsson and Banos, 1995; Guo et al., 2010; Vandenplas et al., 2014). This observation suggests that multi-step methods - that assume independent pseudo-records for sires tested on a large number of progeny with precisely estimated contemporary groups - also do not have an issue of double counting contributions from relatives. The (modified) TSA algorithm (Vandenplas and Gengler, 2012) could therefore be skipped for applications that integrate $(\mathrm{G})$ EBV with high reliability. However, for applications where (G)EBV are based more on relatives' contributions (e.g., sires tested on a smaller number of progeny or genomically predicted from a smaller training population), the method can be used to estimate the amount of double counting and decide whether it is important to avoid it or not. Avoiding double counting of contributions from records did not have high importance in our demonstration. This is because most bulls had highly reliable GEBVs and only 277 national bulls out of the 5,852 bulls with multi-national genomic information contributed to the multi-national genomic evaluation. However, accounting for double counting of contributions from records does not have a high computational cost and we advise using it.

\section{Implications for Breeding Programs}

The developed method; that is, a national ssGBLUP with integration, has at least 3 implications for breeding programs. The method can (1) increase reliability of a national evaluation, (2) combine all available information in one evaluation that gives unified ranking of all animals, and (3) increase the size of an otherwise small national genomic training population.

The method can increase reliability of a national evaluation. If a breeding program participates in some kind of multi-national genomic evaluation (such as InterGenomics or a multinational genomics program run separately by each breeding program), the method allows integrating those additional results back into the initial evaluation, which increases reliability for animals that have limited amount of information in the national breeding program. This is likely to be very important for sires with few phenotyped progeny in the national data set but substantial numbers of phenotyped progeny in other breeding programs. It will also be important for the imported animals or young animals, which will benefit from the increased information coming through pedigree and genomic relationships. However, the increase in reliability for the latter 2 groups of animals will likely be marginal, unless these animals are genotyped. If these animals are not genotyped, the value of 
information on ancestors diminishes very quickly with increasing distance in the pedigree. If these animals are genotyped, the increase in reliability is likely to come from the increased size of the genomic training population (Daetwyler et al., 2008; Goddard, 2009) and increased presence of close relatives in the training population (Clark et al., 2012; Pszczola et al., 2012).The method is also well adapted to increase reliabilities of a national evaluation for novel traits with very limited phenotypic information obtained in small populations with detailed data recording but spread over different places and countries (e.g., Egger-Danner et al., 2015).

The method can combine all available information in one evaluation that gives a unified ranking of all animals. In relation to the previous implication, the ability to perform a national evaluation that uses all national phenotype, pedigree, and genotype data and, in addition, the multi-national genomic information is appealing, because it provides a way to integrate the different evaluations and provide unique ranking of all animals: domestically and internationally proven bulls, cows, as well as young animals. As ssGBLUP blends the pedigree-based and genomic evaluations, our method additionally blends national and multi-national evaluations.

The method can increase the size of the genomic training population. Because integration is performed through the joint pedigree and genomic relationship matrix, the method enables increasing the size of the genomic training population. This critically depends on access to the multi-national pedigree and, more importantly, multi-national genotypes to build such a relationship matrix. In the demonstration, this was the case and we were able to increase the size of the training population from 191 to 5,852, which essentially upgraded the national pedigree-based evaluation with a too-small training population to a national genomic evaluation with a sufficiently large genomic training population. This was not our primary objective, because the breeding programs that participate in the InterGenomics consortium already have a shared multinational genomic evaluation. However, the ability to routinely conduct genomic evaluation in each country in addition to the regular consortium evaluation is a welcome by-product of the method. In other words, the upgraded evaluation can include new genotyped animals by simply extending the relationship matrix.

\section{Other Potential Applications of the Developed Method}

The developed method or its components can be used in other settings that were not studied in detail here.
First, although the method and the demonstration implied that all individuals have multi-national genomic information including genotype data, the method can be applied to more complex situations. For example, the method could be used when some national animals are genotyped but do not have multi-national information (e.g., young animals, cows). Integration of multinational information of nongenotyped individuals can also be performed.

Second, the method can be extended to integrate results of univariate or multivariate multi-national evaluations into national evaluations based on multivariate (random regression) models (Quaas and Zhang, 2006; Vandenplas et al., 2013, 2014, 2015). For situations where we would like to integrate results of different traits from a multivariate multi-national evaluation, contributions from correlated traits must be accounted to avoid double counting when computing $E R C_{i}^{*}$. This can be done by computing $E R C_{i}^{*}$ for all the traits simultaneously using the TSA algorithm modified to mimic the left-hand side of a system of equations of a multivariate evaluation.

The method can also be used for integration of genomic MACE (GMACE) results (Sullivan and VanRaden, 2009) into a national ssGBLUP. The GMACE aggregates national genomic evaluations just like MACE aggregates national pedigree-based evaluations. The GMACE also accounts for the frequent exchange of data between the breeding programs, which is somewhat related to our method. If breeding programs were interested in integrating the GMACE results back into their national genomic evaluations, the developed method could be used. In such a setting, double counting of national information must be avoided, which can be accomplished by modifying the right-hand side of [10] as follows:

$$
\left[\begin{array}{l}
\mathbf{X}^{\prime} \mathbf{R}^{-1} \mathbf{y} \\
\mathbf{Z}^{\prime} \mathbf{R}^{-1} \mathbf{y}+\left(\mathbf{H}^{-1} \sigma_{a}^{2}+\mathbf{\Lambda}^{*}\right) \hat{\mathbf{a}}^{*}-\left(\mathbf{H}^{-1} \sigma_{a}^{2}+\boldsymbol{\Lambda}^{* *}\right) \hat{\mathbf{a}}^{\#}
\end{array}\right],
$$

where $\hat{\mathbf{a}}^{*}$ is the vector of GMACE GEBV for the genotyped animals and of GMACE GEBV predicted for the nongenotyped animals, $\hat{\mathbf{a}}^{\#}$ is a vector of national GEBV obtained from the hypothetical ssGBLUP, and $\boldsymbol{\Lambda}^{* *}$ is a diagonal variance matrix with the diagonal element equal to $R_{i}^{* *} \sigma_{e}^{-2}$ for the $i$ th animal that participated in GMACE. Elements $R_{i}^{* *}$ can be computed using the algorithm of Vandenplas and Gengler (2012) modified for the genomic evaluations as shown in this study.

The modified TSA algorithm could also be used in multi-step methods. The multi-step methods assume 
independent pseudo-records for sires tested on a large number of progeny with precisely estimated contemporary groups in large breeding programs, leading to little effect of double counting contributions from relatives. However, training populations increasingly include genotyped animals with lower reliabilities; for example, cows or sires tested on a smaller number of progeny (e.g., Gao et al., 2015). The (modified) TSA algorithm could be used in such a setting for computation of independent pseudo-records required by the multi-step methods, similarly to Calus et al. (2016).

\section{CONCLUSIONS}

The developed method simultaneously and optimally integrates the results of a multi-national genomic evaluation into a national evaluation that uses phenotype, pedigree, and genotype data. Importantly, it avoids double counting of the dependent data contributions. The resulting evaluation provides consistent and more reliable ranking of all animals: bulls, cows, and young animals. This in turn provides more efficient and transparent selection among all animals.

\section{ACKNOWLEDGMENTS}

Jérémie Vandenplas (as a research fellow) and Nicolas Gengler (as a former senior research associate) acknowledge the support of the National Fund for Scientific Research (Brussels, Belgium) for their positions. The authors also acknowledge financial support from the bilateral cooperation agreement BI-BE/11-12-V-006 between the Wallonie-Bruxelles International (WBI) and Slovenian Research Agency (ARRS), and Institute Strategic Program Grant BB/J004235/1 of the Biotechnology and Biological Sciences Research Council (UK). The authors acknowledge the use of data from the InterGenomics consortium. This work has made use of the resources provided by the Consortium des Equipements de Calcul Intensif (CECI), funded by the National Fund for Scientific Research (Belgium) under Grant No. 2.5020.11. Useful comments from the two anonymous reviewers are acknowledged.

\section{REFERENCES}

Aguilar, I., I. Misztal, D. L. Johnson, A. Legarra, S. Tsuruta, and T. J. Lawlor. 2010. Hot topic: A unified approach to utilize phenotypic, full pedigree, and genomic information for genetic evaluation of Holstein final score. J. Dairy Sci. 93:743-752.

Calus, M. P. L., J. Vandenplas, J. ten Napel, and R. F. Veerkamp. 2016. Validation of simultaneous deregression of cow and bull breeding values and derivation of appropriate weights. J. Dairy Sci. https://doi.org/10.3168/jds.2016-11028.
Clark, S. A., J. M. Hickey, H. D. Daetwyler, and J. H. van der Werf. 2012. The importance of information on relatives for the prediction of genomic breeding values and the implications for the makeup of reference data sets in livestock breeding schemes. Genet. Sel. Evol. 44:4.

Colinet, F. G., J. Vandenplas, P. Faux, S. Vanderick, R. Renaville, C. Bertozzi, X. Hubin, and N. Gengler. 2013. Walloon single-step genomic evaluation system integrating local and MACE EBV. Interbull Bull. 47:203-210.

Daetwyler, H. D., B. Villanueva, and J. A. Woolliams. 2008. Accuracy of predicting the genetic risk of disease using a genome-wide approach. PLoS One 3:e3395.

Egger-Danner, C., J. B. Cole, J. E. Pryce, N. Gengler, B. Heringstad, A. Bradley, and K. F. Stock. 2015. Invited review: Overview of new traits and phenotyping strategies in dairy cattle with a focus on functional traits. Animal 9:191-207.

Gao, H., P. Madsen, U. S. Nielsen, G. P. Aamand, G. Su, K. Byskov, and J. Jensen. 2015. Including different groups of genotyped females for genomic prediction in a Nordic Jersey population. J. Dairy Sci. 98:9051-9059.

Goddard, M. 2009. Genomic selection: Prediction of accuracy and maximisation of long term response. Genetica 136:245-257.

Guo, G., M. s. Lund, Y. Zhang, and G. Su. 2010. Comparison between genomic predictions using daughter yield deviation and conventional estimated breeding value as response variables. J. Anim. Breed. Genet. 127:423-432.

Henderson, C. R. 1984. Applications of Linear Models in Animal Breeding. 2nd ed. University of Guelph, Guelph, ON, Canada.

Jorjani, H., J. Jakobsen, E. Hjerpe, V. Palucci, and J. Dürr. 2012. Status of genomic evaluation in the Brown Swiss populations. Interbull Bull. 46:46-54.

Legarra, A., J. K. Bertrand, T. Strabel, R. L. Sapp, J. P. Sanchez, and I. Misztal. 2007. Multi-breed genetic evaluation in a Gelbvieh population. J. Anim. Breed. Genet. 124:286-295.

Legarra, A., O. F. Christensen, I. Aguilar, and I. Misztal. 2014. Single Step, a general approach for genomic selection. Livest. Sci. 166:54-65.

Lund, M. S., A. P. W. de Roos, A. G. de Vries, T. Druet, V. Ducrocq, S. Fritz, F. Guillaume, B. Guldbrandtsen, Z. Liu, R. Reents, C. Schrooten, M. Seefried, and G. Su. 2010. Improving genomic prediction by EuroGenomics collaboration. Proc. 9th World Congress on Genetics Applied Livestock Production (WCGALP), Leipzig, Germany. German Society for Animal Science, Neustadt, Germany.

Misztal, I. 2013. BLUPF90 family of programs. University of Georgia, Athens. Accessed Jan. 15, 2013. http://nce.ads.uga.edu/wiki/ doku.php.

Misztal, I., and G. R. Wiggans. 1988. Approximation of prediction error variance in large-scale animal models. J. Dairy Sci. 71(Suppl. 2):27-32

Potočnik, K., M. Kovač, and J. Pogačar. 2000. Estimation of dispersion parameters with an animal test-day model for Slovenian black and white breed. Acta Agric. Slov. 76:49-60.

Přibyl, J., P. Madsen, J. Bauer, J. Přibylová, M. Šimečková, L. Vostrý, and L. Zavadilová. 2013. Contribution of domestic production records, Interbull estimated breeding values, and single nucleotide polymorphism genetic markers to the single-step genomic evaluation of milk production. J. Dairy Sci. 96:1865-1873.

Pszczola, M., T. Strabel, H. A. Mulder, and M. P. L. Calus. 2012. Reliability of direct genomic values for animals with different relationships within and to the reference population. J. Dairy Sci. 95:389-400.

Quaas, R. L., and Z. Zhang. 2006. Multiple-breed genetic evaluation in the US beef cattle context: Methodology. CD-Rom Commun. 24-12 in Proc. 8th World Congress on Genetics Applied to Livestock Production. Belo Horizonte, Brazil. Instituto Prociência, Minas Gerais, Brazil.

Schaeffer, L. R. 1994. Multiple-country comparison of dairy sires. J. Dairy Sci. 77:2671-2678. 
Sigurdsson, A., and G. Banos. 1995. Dependent variables in international sire evaluations. Acta Agric. Scand. A Anim. Sci. 45:209 217.

Sullivan, P. G., and P. M. VanRaden. 2009. Development of genomic GMACE. Interbull Bull. 40:157-161.

Vandenplas, J., F. G. Colinet, and N. Gengler. 2014. Unified method to integrate and blend several, potentially related, sources of information for genetic evaluation. Genet. Sel. Evol. 46:59.

Vandenplas, J., F. G. Colinet, G. Glorieux, C. Bertozzi, and N. Gengler. 2015. Integration of external estimated breeding values and associated reliabilities using correlations among traits and effects. J. Dairy Sci. 98:9044-9050.

Vandenplas, J., and N. Gengler. 2012. Comparison and improvements of different Bayesian procedures to integrate external information into genetic evaluations. J. Dairy Sci. 95:1513-1526.
Vandenplas, J., S. Janssens, N. Buys, and N. Gengler. 2013. An integration of external information for foreign stallions into the Belgian genetic evaluation for jumping horses. J. Anim. Breed. Genet. 130:209-217.

VanRaden, P. M. 2008. Efficient methods to compute genomic predictions. J. Dairy Sci. 91:4414-4423.

VanRaden, P. M., M. E. Tooker, J. R. Wright, C. Sun, and J. L. Hutchison. 2014. Comparison of single-trait to multi-trait national evaluations for yield, health, and fertility1. J. Dairy Sci. 97:79527962

VanRaden, P. M., C. P. Van Tassell, G. R. Wiggans, T. S. Sonstegard, R. D. Schnabel, J. F. Taylor, and F. S. Schenkel. 2009. Invited Review: Reliability of genomic predictions for North American Holstein bulls. J. Dairy Sci. 92:16-24. 